\title{
ANÁLISE EXPLORATÓRIA DAS CARACTERÍSTICAS MORFOLÓGICAS E QUALITATIVAS DE VARIEDADES DE LARANJEIRAS DE MESA DA COLEÇÃO EM BREJÃO - PE ${ }^{1}$
}

RENATA CRISTINA MEDEIROS ${ }^{2}$, ROSIMAR DOS SANTOS MUSSER ${ }^{3}$, MAIRON MOURA DA SILVA $^{4}$ JOSÉ PEROBA OLIVEIRA SANTOS ${ }^{5}$, IVANILDO RAMALHO DO NASCIMENTO JÚNIOR ${ }^{6}$

RESUMO- Este trabalho teve como objetivo avaliar as características morfológicas e os aspectos físicos, físico-químicos e químicos das plantas e dos frutos de 15 variedades de laranjas cedidas pelo Centro Nacional de Pesquisa de Mandioca e Fruticultura (Embrapa - CNPMF), e plantadas na Estação Experimental do Instituto Agronômico de Pernambuco, Brejão-PE. Foram avaliados: diâmetro do caule acima e abaixo da linha de enxertia e a relação entre eles, diâmetro da copa, altura da planta e volume de copa. Nos frutos, as avaliações consistiram em: formato, coloração do epicarpo, massa do fruto, massa do suco, altura e diâmetro do fruto, espessura do mesocarpo e do endocarpo, número de sementes por fruto e rendimento em suco. O delineamento experimental foi em blocos casualisados, com quatro repetições e uma planta por parcela. Para o aspecto qualitativo dos frutos, utilizou-se o mesmo delineamento, e foram avaliados através de 12 descritores. Apenas seis das variedades de laranjas estudadas estão dentro das exigências do Programa Brasileiro para a Modernização da Horticultura e cinco das variedades de laranjas mostraram-se com maturação diferente das descritas na literatura.

Termos para indexação: Coleção, desenvolvimento, análises físico-química.

\section{EXPLORATORY ANALYSIS OF MORPHOLOGICAL AND QUALITATIVE CHARACTERISTICS OF ORANGE VARIETIES IN BREJÃO-EP COLLECTION}

\begin{abstract}
Studies on citrus table fruits still has little expression in the country. This study aimed to analyze the morphological and the physical, physico-chemical and chemical characteristics of plants and fruits of 15 varieties of oranges, courtesy of the National Research Center for Cassava and Fruit (Embrapa CNPMF), and planted at the Experimental Station of the Agronomic Institute of Pernambuco, Brejão-PE. It was evaluated: stem diameter above and below the graft and the relationship between them, crown diameter, plant height and canopy volume. In fruits evaluations consisted of: format, epicarp color, fruit weight and height, juice weight, , fruit diameter, thickness of mesocarp and endocarp, number of seeds per fruit and juice yield. The experimental design was randomized blocks with four replications and one plant per plot. For the qualitative aspect of the fruits it was used the same design, they were evaluated by 12 descriptors. Only six of the studied varieties of oranges are within the requirements of the Brazilian Program for the Modernization of Horticulture and five varieties of oranges showed different maturation from those described in the literature Index terms: collection, development, analysis physical chemistry.
\end{abstract}

\footnotetext{
'(Trabalho 227-12). Recebido em: 27-08-2012. Aceito para publicação em: 02-05-2013.

${ }^{2}$ Engenheira Agrônoma, Mestranda em Agronomia - Melhoramento Genético de Plantas - Universidade Federal Rural de Pernambuco (UFRPE). Email: renatamedeiros@agronoma.eng.br.

${ }^{3}$ Doutora, Professora Associada do Departamento de Agronomia da Universidade Federal Rural de Pernambuco (UFRPE). Email: rosimar.musser@gmail.com

${ }^{4}$ Doutor, Professor Adjunto da Unidade Acadêmica de Garanhuns (UAG-UFRPE). Email: maironmoura@uag.ufrpe.br

${ }^{5}$ Mestre, Pesquisador do Instituto Agronômico de Pernambuco (IPA), Email: perobaoliveira@yahoo.com.br

${ }^{6}$ Engenheiro Agrônomo, Mestrando em Agronomia - Melhoramento Genético de Plantas - Universidade Federal Rural de Pernambuco (UFRPE). E-mail: ivanildoramalho@gmail.com
} 


\section{INTRODUÇÃO}

O Brasil, com seu vasto território, caracterizado com alterações climáticas e solo bastante diversificado, destaca-se mundialmente na produção de laranja para extração de suco. Os citros geram em torno de US\$ 2,5 bilhões por ano (NEVES et al., 2010), ocupando o primeiro lugar em cultivo e produção (FAO, 2012). Os principais citros cultivados no País são as laranjeiras, tangerineiras, limeiras-ácidas e os limões-verdadeiros (NEVES et al., 2010). Os laranjais, distribuídos em todo o território brasileiro, destacam-se nos Estados de São Paulo, Bahia, Sergipe, entre outros, com menor potencial, que, juntos, em 2009, produziram cerca de 18 milhões de toneladas de frutas frescas (IBGE, 2012).

A maior parte da produção brasileira é destinada à industrialização e produção de suco, principalmente o concentrado e congelado (FCOJ), com aproximadamente 1,1 milhão de toneladas em 2009. Já a produção de frutos para a comercialização in natura obteve, no mesmo período, aproximadamente 26 milhões de $\mathrm{kg}$ de frutas frescas (NEVES et al., 2010). A quantidade em $\mathrm{kg}$ de frutas frescas para a extração de FCOJ, de acordo com Munhoz et al. (2010), deve-se à sazonalidade e à estabilidade na demanda dos produtos, que resulta em ajustes do teor da polpa do produto dentro dos padrões desejados.

A produção dos frutos e sua comercialização estão ligadas a diversos fatores, entre os quais, as características morfológicas dos frutos, que podem sofrer variações em função dos caracteres ambientais, (MACHADO, et al.) idade da planta, ocorrência de patógenos sistêmicos e do variado manejo sofrido, o fruto, em particular, apresenta alterações na cor do epicarpo e do suco, no teor de sólidos solúveis, na espessura do mesocarpo, no número de sementes, dentre outros aspectos (DONADIO et al., 2005). A qualidade interna e externa dos frutos para consumo in natura são prioridades na produção de frutas cítricas Stuchi et al. (2002), revisando trabalhos sobre produção e os aspectos quantitativos, percebe-se, que geralmente se referiam às características diretas de qualidade quanto à forma, tamanho e ao peso dos frutos, sólidos solúveis totais, acidez e rendimento em suco.

Estudos que envolvam o desenvolvimento e a produção de frutos de citros e suas diversas variedades de copas com seus porta-enxertos em diferentes condições ambientais podem levar a uma melhora nas práticas de manejo em citros (FIDALSKI et al., 2007).

O objetivo do presente trabalho de pesquisa foi analisar as características morfológicas das plan- tas e os aspectos físicos, físico-químicos e químicos dos frutos de 15 variedades comerciais de laranjas cedidas pelo Centro Nacional de Pesquisa de Mandioca e Fruticultura (Embrapa-CNPMF), que fazem parte do seu programa de melhoramento genético em estudo. Estas foram plantadas no Agreste Meridional de Pernambuco.

\section{MATERIAL E MÉTODOS}

A coleção de variedades comerciais de citros estudados possui cinco anos de idade, conduzida na Estação Experimental do Instituto Agronômico de Pernambuco (IPA), no município de Brejão-PE. A microrregião faz parte do Agreste Meridional de Pernambuco ( $8^{\circ} 53^{\prime} 44^{\prime \prime} \mathrm{S}$, longitude $36^{\circ} 31^{\prime} 26^{\prime \prime}$ $\mathrm{W}$, altitude média de $820 \mathrm{~m}$ ). O clima caracteriza-se como seco e subúmido, com temperatura média mensal que varia de 17 a $22{ }^{\circ} \mathrm{C}$ ao longo do ano e precipitação média anual de 782,4 mm (SECRETARIA DE CIÊNCIA, TECNOLOGIA E MEIO AMBIENTE DE PERNAMBUCO, 2006; INMET, 2012). Os dados mensais de precipitação e temperatura do ar, entre os meses de agosto de 2010 e agosto de 2011 do município, foram cedidos pela Agência Pernambucana de Águas e Clima (APAC,2012), filiada à Secretaria de recursos hídricos e energéticos do Estado de Pernambuco.

Foram avaliadas 15 variedades de laranjeiras de cinco anos de idade, plantadas em espaçamento de 6x4 m: Bahia 101; Baianinha 03 sobre o porta-enxerto CLEO-SW-264; Hamlin 02, Lima, Rubi, Sunstar, Pineapple, Pera D6, Westin, Midsweet, Salustiana sobre o porta-enxerto LVK-CLEO-264; Pera D12, Valência Tuxpan, sobre o porta-enxerto LVK-CLEO-226; Natal sobre o porta-enxerto LCR-CLEO-256; Pera D9 sobre o porta-enxerto LCR-LVK-CLEO. O sistema de irrigação utilizado foi por microaspersão, empregado conforme as condições de umidade do solo.

Levando-se em consideração que o principal objetivo da pesquisa é a qualidade dos frutos, a análise de crescimento das plantas é um complemento para a investigação. $\mathrm{O}$ delineamento experimental adotado foi em blocos ao acaso, com quatro repetições por variedade, uma planta por parcela, sendo quatro frutos por planta, quantidade esta dentro do indicado por STENZEL, 2005. Foram realizadas duas medições para avaliação do crescimento vegetativo, nos meses de junho/2010 e junho/2011. Nestas datas, foram medidos: circunferência do caule, $5 \mathrm{~cm}$ abaixo da enxertia e $5 \mathrm{~cm}$ acima da enxertia $(\mathrm{cm})$; altura da planta, tomada desde o solo até o topo da copa $(\mathrm{m}) \mathrm{e}$ o diâmetro da copa, que foi medido com uma régua 
graduada em $\mathrm{cm}$ no sentido paralelo e perpendicular à linha de plantio, segundo a metodologia descrita por Stenzel et al. (2005).

Com medidas da circunferência do caule, foi calculado o raio do caule e o índice de compatibilidade entre copa e porta-enxerto, seguindo a metodologia descrita por Negreiros et al. (2010). O volume da copa foi calculado com os dados de altura e diâmetro da planta, segundo a proposta de Mendel (1956) e descrita por Stenzel et al. (2005).

As avaliações do período de colheita foram realizadas entre os meses de outubro de 2010 e agosto de 2011. No mês de outubro de 2010, foram marcados quatro ramos que apresentavam flores abertas nos quatro quadrantes da copa, em quatro plantas de cada variedade. Foi tomado como base o período da antese, em que a maioria das plantas possuía flores abertas, até o ponto de colheita (SACRAMENTO et al., 1988), descrita pela escala fenológica da $\mathrm{BBCH}$ entre os estádios de 6 a 8 (AGUSTI et al, 1997). Posteriormente, calculou-se o número acumulado de graus-dia (GD) correspondente à data e ao mês do período considerado. Para o cálculo de GD acumulado, utilizou-se a fórmula segundo Pereira et al. (2006), em que GD = ((Tmáx - Tmín ) / 2 ) - Tb, sendo GD a soma térmica em graus-dia, Tmáx a temperatura máxima, Tmín a temperatura mínima e $\mathrm{Tb}$ a temperatura base adotada para os citros $\left(13^{\circ} \mathrm{C}\right)$.

Mensalmente, desde o início da maturação dos frutos, de fevereiro a agosto de 2011, foram colhidos quatro frutos por parcela nos quatro quadrantes da planta, na parte externa e mediana da copa, segundo metodologia adaptada por Stenzel et al. (2005). As amostras foram acondicionadas em bolsas apropriadas e levadas para o Laboratório de Biologia Vegetal da UFRPE-UAG, em Garanhuns. Estes frutos foram utilizados para as análises físicas, físico-químicas e químicas.

Nas amostras, foram determinados: formato, com a metodologia proposta IPGRI (1999); coloração do epicarpo, segundo Schinor et al. (2009); massa do fruto $(\mathrm{g})$; massa do suco $(\mathrm{g})$; altura, aferida do pedúnculo ao ápice, e diâmetro do fruto ( $\mathrm{mm}$ ); espessura do mesocarpo e endocarpo $(\mathrm{mm})$; número de sementes por fruto; e rendimento em suco (\%) (STENZEL et al., 2005). As medições internas da espessura do epicarpo, mesocarpo e endocarpo foram em quatro pontos distintos e correspondentes, perfazendo a circunferência do fruto. O rendimento de suco foi obtido após esmagamento em extratora manual, calculado por meio da relação massa do suco/massa do fruto e expresso em porcentagem. Em seguida, foi realizada a contagem de sementes.

As avaliações físico-químicas e químicas foram: sólidos solúveis, expressos em ${ }^{\circ}$ Brix, acidez titulável, expressa em \% de ácido cítrico, teor de vitamina $\mathrm{C}$ (ácido ascórbico) e pH. As amostras de suco foram colocadas em freezer por, no máximo, cinco dias para posterior determinação de sólidos solúveis (SS), realizada com leitura direta em refratômetro manual, sendo o resultado expresso em ${ }^{\circ} \mathrm{Brix}$, corrigidos pela temperatura do suco. O método de titulação empregado seguiu a metodologia descrita pelo Official Methods of Analysis of Internacional (AOAC, 2005), sendo utilizados $25 \mathrm{~mL}$ de suco com solução de hidróxido de sódio $(\mathrm{NaOH})$ a $0,1 \mathrm{~N}$. A concentração de vitamina $C$ (ácido ascórbico) foi determinada pelo método titulométrico do 2,6-diclorofenolindofenol (AOAC, 2005). A determinação do $\mathrm{pH}$ foi realizada por meio de método direto em potenciômetro, devidamente calibrado e higienizado.

Os dados obtidos foram processados e submetidos à análise de variância, e as médias, comparadas através do teste de Scott- Knott, a 1\% de probabilidade, com o auxílio do programa GENES da Universidade Federal de Viçosa (UFV).

\section{RESULTADOS E DISCUSSÃO}

Todas as variedades de laranjeira estudadas apresentaram crescimento em tamanho no período avaliado (Tabelas 1 e 2). A Variedade Natal destacou-se em altura, em contraposição ao clone Pera D6, que teve o menor crescimento. Segundo Westephalen (2008), o porte da planta deve ser considerado como um requisito de influência no manuseio da colheita, facilitando este processo, e Corrêa et al. (2009) descrevem que a colheita deve ser uma atividade que proporcione menos injúrias aos frutos, desconforto físico e maior segurança ao agricultor.

No volume da copa (Tabela 2), a laranjeira Valência Tuxpan destacou-se por apresentar, em julho de 2011, o maior volume, com 19,67 $\mathrm{m}^{3}$. As variedades Pera D6, Westin e Midsweet apresentaram menor volume de copa (Tabela 2), característica esta que traz vantagens ao citricultor, proporcionando maior facilidade no manejo da planta em relação ao controle das pragas e doenças, e facilidade na colheita. $\mathrm{O}$ índice de compatibilidade entre enxerto/ porta-enxerto mostra que todas as variedades estudadas se encontraram próximas ao valor favorável citado por Negreiros et al. ( 2010) (Tabelas 1 e 2).

Em relação aos aspectos físicos dos frutos, verificou-se que a maior altura do fruto $(\mathrm{AF})$ foi na variedade Baianinha 03 , com média de $77,76 \mathrm{~mm}$; entretanto, nota-se que o maior diâmetro transversal de fruto (DTF) foi a variedade Valência Tuxpan, 
com 82,58 mm (Tabela 3). Conforme os parâmetros citados por Schinor et al. (2009), todas as variedades estudadas apresentaram dimensões satisfatórias e dentro do padrão do Programa Brasileiro para Modernização da Horticultura (2011).

Quanto à massa dos frutos (MF), a laranjeira variedade Natal destacou-se com média de $260 \mathrm{~g}$ (Tabela 3). De acordo com Domingues et al. (2003), para o mercado de laranjas in natura, os frutos devem apresentar o mínimo de $150 \mathrm{~g}$; mas aqui é necessário destacar que a laranjeira- Lima foi a única variedade que não atendeu a este padrão, mostrando menor MF.

O quanto ao rendimento de suco (RS), destacou-se que todas as variedades de laranjeira apresentaram valores compatíveis e acima do mínimo exigido pelo Programa Brasileiro para Modernização da Horticultura (2011), que é de 35\%, com destaque entre as laranjeiras para a variedade Salustiana, que apresentou RS de $61 \%$ (Tabela 3 ).

Oliveira et al. (2010) mencionam que os consumidores manifestam preferência por frutos com casca mais fina, para facilitar o descascamento; neste parâmetro, destacou-se a variedade Salustiana como de menor espessura de epicarpo mais mesocarpo (EPEM), sendo de 3,20 mm (Tabela 3). A variedade Sunstar apresentou maior EPEM, com 55,37 mm. Já para a espessura do endocarpo (EEN), o maior valor foi para a variedade Pera D9 com 422,37 mm e o menor para a variedade de laranja-Lima com 22,69 mm (Tabela 3). Quanto ao formato do fruto, ocorreram apenas dois formatos, o elipsoide e o esferoide, de acordo com o IPGRI (1999) (Tabela 3).

A variedade Midsweet foi a que apresentou, em média, maior número de semente (NS) com 12,75 , e o resultado revelado foi acima do citado para a variedade nas condições edafoclimáticas de Cruz das Almas, na Bahia (POMPEU JÚNIOR et al., 2009) (Tabela 3).

As variedades Bahia 101 e Baianinha 03 não apresentaram sementes, enquadrando-se nos termos citados por Sartori et al. (2002) e Passos et al. (2006) por serem variedades partenocárpicas. Vale ressaltar que uma das características desejadas pelos consumidores de frutos in natura é o reduzido número de sementes (PEREIRA et al., 2006; OLIVEIRA et al., 2010).

Para o teor de vitamina $C$, não foram observadas diferenças significativas entre as variedades, porém o coeficiente de variação mostrou-se elevado. No entanto, os teores médios observados foram similares aos relatados por outros autores (PELLEGRINE et al., 2007; COUTO; CANIATTI-BRAZACA, 2010).

A laranja Bahia 101 destacou-se, apresentando nível de sólidos solúveis (SS) de $10,35^{\circ}$
Brix (Tabela 4). Diante das variedades estudadas, percebeu-se que apenas as laranjas Baianinha 03, Hamlin 02, Lima, Rubi, Pineapple, Pera D6, Pera D9, Valência Tuxpan, Midsweet e Westin se encontram dentro dos teores descritos por Pereira et al. (2006) e mais próximos do exigido pelo Programa Brasileiro para Modernização da Horticultura (2011); as demais variedades posicionaram-se pouco abaixo do padrão mínimo recomendado, que é entre 9 e $10^{\circ}$ Brix.

Diante do exposto por Pereira et al. (2006), a acidez titulável (AT) das laranjas maduras devem estar entre 0,5 e $1 \%$ de ácido cítrico, e de acordo com o Programa Brasileiro para Modernização da Horticultura (2011), a AT é de 1,05. Dentro do revelado, infere-se dizer que todas as variedades analisadas se encontraram dentro desta porcentagem, com exceção das variedades Bahia 101, Rubi, Pera D9 e Valência Tuxpan (Tabela 4).

Em relação ao teor de vitamina $\mathrm{C}$ (ácido ascórbico), destacou-se a variedade Pera D9, com teor de ácido ascórbico (a.a.) de 69,71 mg/100 mg de suco;em contraposição, encontra-se a Hanlim 02 com 25,16 mg/100 mg de suco. O teor de vitamina C (a. a.), de acordo com Donadio et al. (2005) em citros, altera-se de acordo com a variedade e o grau de amadurecimento.

A relação SS/AT é considerada por Sartori et al. (2002) como o indicador de maturação dos frutos e relataram que, para o consumo in natura, os frutos de laranjeiras devem apresentar razão de ST/AT acima de 8. Diante dos resultados obtidos, verifica-se que todas as variedades de laranjas são adequadas para o consumo, exceto a Valência Tuxpan, que apresentou média abaixo do indicado (Tabela 4). As variedades Rubi, Sunstar, Pera D9 e Valência Tuxpan apresentaram razão de ST/AT abaixo do mínimo exigido pelo Programa Brasileiro para Modernização da Horticultura (2011), não atingindo assim o padrão de qualidade.

Quanto à maturação das laranjas, foi observado que as variedades Pineapple e Valência Tuxpan se diferenciaram do descrito por Pompeu Júnior (2005) e Passos et al. (2006), alcançando maturação com menor GD acumulado, enquadrando-se em plantas de maturação de meia-estação. Este fator pode ter-se dado por diversos fatores, entre os quais a idade das plantas e as condições climáticas na época de avaliação. Notou-se que as variedades Sunstar e Natal, descritas por Pompeu Júnior (2009) e Souza et al. (2010) como variedades de maturação tardia, nas condições do presente estudo, comportaram-se como variedades de maturação em meia- estação. 
TABELA 1- Médias numéricas de crescimento vegetativo de 15 variedades de citros da coleção de BrejãoPE, junho de 2010.

\begin{tabular}{lcccccc}
\hline \multicolumn{1}{c}{ Variedades } & $\begin{array}{c}\text { D. Ac. } \\
(\mathbf{c m})\end{array}$ & $\begin{array}{c}\text { D. Ab. } \\
\mathbf{( c m )}\end{array}$ & $\begin{array}{c}\text { R. C. } \\
\mathbf{( m )}\end{array}$ & $\begin{array}{c}\text { Altura da Planta } \\
(\mathbf{m})\end{array}$ & $\begin{array}{c}\text { Volume de copa } \\
\left(\mathbf{m}^{3} \mathbf{)}\right.\end{array}$ & $\begin{array}{c}\text { R. En./P. Em. } \\
(\mathbf{c m})\end{array}$ \\
\hline Laranjas & Média & Média & Média & Média & Média & Média \\
Bahia 101 & 12,02 & 14,29 & 1,52 & 2,89 & 14,54 & 1,24 \\
Baianinha 03 & 11,64 & 13,46 & 1,47 & 2,21 & 10,21 & 1,25 \\
Hamlin 02 & 13,89 & 14,97 & 1,45 & 2,68 & 11,98 & 1,08 \\
Lima & 12,73 & 13,70 & 1,36 & 2,62 & 10,34 & 1,07 \\
Rubi & 11,86 & 15,09 & 1,47 & 2,34 & 11,19 & 1,29 \\
Sunstar & 12,37 & 14,84 & 1,49 & 2,68 & 12,56 & 1,20 \\
Pineapple & 11,72 & 14,50 & 1,42 & 2,98 & 12,63 & 1,24 \\
Pera D6 & 11,90 & 13,24 & 1,44 & 2,16 & 9,55 & 1,13 \\
Pera D9 & 11,51 & 12,25 & 1,49 & 2,44 & 11,75 & 1,06 \\
Pera D 12 & 12,67 & 13,18 & 1,60 & 2,90 & 15,89 & 1,05 \\
Valência Tuxpan & 13,07 & 15,18 & 1,71 & 3,16 & 19,67 & 1,17 \\
Westin & 12,90 & 14,46 & 1,36 & 2,52 & 9,76 & 1,14 \\
Midsweet & 11,37 & 14,29 & 1,25 & 2,80 & 9,60 & 1,27 \\
Natal & 14,39 & 15,35 & 1,61 & 3,23 & 17,68 & 1,07 \\
Salustiana & 13,27 & 15,48 & 1,66 & 2,84 & 16,41 & 1,17 \\
\hline
\end{tabular}

D.Ac.: Diâmetro acima da linha de enxertia; D.Ab.: Diâmetro abaixo da linha de enxertia; R.C.: Raio da Copa; R. En / P. En: Relação enxerto/Porta-enxerto.

TABELA 2 - Médias numéricas de crescimento vegetativo de 15 variedades de citros da coleção de Brejão-PE, junho de 2011.

\begin{tabular}{lcccccc}
\hline Variedades & $\begin{array}{c}\text { D. Ac. } \\
\mathbf{( c m})\end{array}$ & $\begin{array}{c}\text { D. Ab. } \\
\mathbf{( c m )}\end{array}$ & $\begin{array}{c}\text { R. C. } \\
\mathbf{( m )}\end{array}$ & $\begin{array}{c}\text { Altura da Planta } \\
\mathbf{( m )}\end{array}$ & $\begin{array}{c}\text { Volume de copa } \\
\left(\mathbf{m}^{\mathbf{3}}\right)\end{array}$ & $\begin{array}{c}\text { R. En./P. Em. } \\
\mathbf{( c m})\end{array}$ \\
\hline Laranjas & Média & Média & Média & Média & Média & Média \\
Bahia 101 & 10,84 & 13,05 & 1,23 & 2,39 & 7,72 & 1,23 \\
Baianinha 03 & 9,45 & 11,63 & 1,21 & 2,16 & 6,75 & 1,23 \\
Hamlin 02 & 12,35 & 13,6 & 1,29 & 2,54 & 9,05 & 1,10 \\
Lima & 10,61 & 12,17 & 1,24 & 2,41 & 8,04 & 1,16 \\
Rubi & 10,77 & 13,04 & 1,28 & 2,14 & 7,5 & 1,21 \\
Sunstar & 11,55 & 13,84 & 1,24 & 2,61 & 8,75 & 1,20 \\
Pineapple & 10,84 & 12,61 & 1,19 & 2,47 & 7,41 & 1,17 \\
Pera D6 & 10,67 & 11,62 & 1,21 & 2,12 & 6,93 & 1,09 \\
Pera D9 & 10,70 & 11,24 & 1,08 & 2,42 & 6,38 & 1,05 \\
Pera D 12 & 11,44 & 11,76 & 1,37 & 2,74 & 11,15 & 1,03 \\
Valência Tuxpan & 12,22 & 14,02 & 1,41 & 2,85 & 12,29 & 1,16 \\
Westin & 11,42 & 12,87 & 1,26 & 2,47 & 8,27 & 1,13 \\
Midsweet & 10,70 & 13,54 & 1,00 & 2,37 & 5,02 & 1,27 \\
Natal & 13,26 & 13,88 & 1,4 & 2,76 & 11,43 & 1,05 \\
Salustiana & 12,25 & 13,48 & 1,38 & 2,70 & 10,79 & 1,10 \\
\hline
\end{tabular}

D.Ac.: Diâmetro acima da linha de enxertia; D.Ab.: Diâmetro abaixo da linha de enxertia; R.C.:Raio da Copa; R. En / P. En: Relação enxerto/Porta-enxerto. 
TABELA 3- Médias das análises físicas dos frutos da coleção de citros em Brejão -PE, 2011.

\begin{tabular}{|c|c|c|c|c|c|c|c|c|c|c|}
\hline Variedades & $\begin{array}{l}\text { MF } \\
\text { (g) }\end{array}$ & $\begin{array}{l}\text { MS } \\
\text { (g) }\end{array}$ & $\begin{array}{l}\text { R S } \\
(\%)\end{array}$ & NS & $\begin{array}{l}\text { DTF } \\
(\mathrm{mm})\end{array}$ & $\begin{array}{c}\mathbf{A F} \\
(\mathrm{mm})\end{array}$ & $\begin{array}{c}\text { EPEM } \\
(\mathrm{mm})\end{array}$ & $\begin{array}{l}\text { EEN } \\
(\mathrm{mm})\end{array}$ & CEP & FF \\
\hline Laranjas & $\mathbf{M}+\mathbf{G}$ & $\mathbf{M}+\mathbf{G}$ & $\mathbf{M}+\mathbf{G}$ & $\mathbf{M}+\mathbf{G}$ & $\mathbf{M}+\mathbf{G}$ & $\mathbf{M}+\mathbf{G}$ & Médias & Médias & $\mathbf{M}+\mathbf{G}$ & \\
\hline Bahia 101 & $230 a$ & $110 \mathrm{a}$ & $47 \mathrm{~b}$ & $0,00 \mathrm{c}$ & $76,44 \mathrm{a}$ & $74,70 \mathrm{a}$ & 42,12 & 29,91 & $2,25 \mathrm{a}$ & 1 \\
\hline Baianinha 03 & $240 \mathrm{a}$ & $140 \mathrm{a}$ & $55 \mathrm{a}$ & $0,00 \mathrm{c}$ & $76,51 \mathrm{a}$ & $77,76 \mathrm{a}$ & 41,62 & 28,23 & $2,50 \mathrm{a}$ & 1 \\
\hline Hamlin 02 & $250 \mathrm{a}$ & $120 \mathrm{a}$ & $50 \mathrm{~b}$ & $4,75 \mathrm{~b}$ & $77,03 \mathrm{a}$ & $77,67 \mathrm{a}$ & 45,62 & 265,97 & $1,20 \mathrm{a}$ & 1 \\
\hline Lima & $110 \mathrm{~b}$ & $50 \mathrm{~b}$ & $44 \mathrm{~b}$ & $4,50 \mathrm{~b}$ & $61,09 b$ & $58,93 b$ & 4,23 & 22,69 & $2,00 \mathrm{a}$ & 1 \\
\hline Rubi & $170 \mathrm{~b}$ & $80 \mathrm{~b}$ & $49 \mathrm{~b}$ & $13,00 \mathrm{a}$ & $68,8 \mathrm{~b}$ & $62,96 \mathrm{~b}$ & 4,85 & 25,14 & $1,37 \mathrm{a}$ & 1 \\
\hline Sunstar & $220 \mathrm{a}$ & $100 \mathrm{a}$ & $51 \mathrm{~b}$ & $11,25 \mathrm{a}$ & $76,47 \mathrm{a}$ & $71,10 \mathrm{a}$ & 55,37 & 27,19 & $1,50 \mathrm{a}$ & 1 \\
\hline Pineapple & $210 a$ & $110 \mathrm{a}$ & $54 \mathrm{a}$ & $12,50 \mathrm{a}$ & $75,75 \mathrm{a}$ & $66,20 \mathrm{~b}$ & 3,85 & 289,22 & $4,37 \mathrm{a}$ & 1 \\
\hline Pera D6 & $150 \mathrm{~b}$ & $90 \mathrm{~b}$ & $56 a$ & $5,25 \mathrm{~b}$ & $65,87 \mathrm{~b}$ & $64,25 \mathrm{~b}$ & 40,87 & 32,24 & $2,12 \mathrm{a}$ & 2 \\
\hline Pera D9 & $190 \mathrm{~b}$ & $110 \mathrm{a}$ & $54 \mathrm{a}$ & $5,50 \mathrm{~b}$ & $70,94 b$ & $70,85 a$ & 4,50 & 422,37 & $2,12 \mathrm{a}$ & 2 \\
\hline Pera D 12 & $180 \mathrm{a}$ & $110 \mathrm{a}$ & $58 \mathrm{a}$ & $6,00 \mathrm{~b}$ & $70,18 b$ & $68,15 b$ & 44,77 & 330,97 & $2,25 \mathrm{a}$ & 2 \\
\hline Valência Tuxpan & $240 \mathrm{a}$ & $130 \mathrm{a}$ & $59 \mathrm{a}$ & $6,75 \mathrm{~b}$ & $82,58 \mathrm{a}$ & $74,64 \mathrm{a}$ & 32,87 & 305,47 & $1,37 \mathrm{a}$ & 1 \\
\hline Westin & $200 \mathrm{a}$ & $120 \mathrm{a}$ & $58 \mathrm{a}$ & $4,75 \mathrm{~b}$ & $74,08 \mathrm{a}$ & $68,42 b$ & 43,92 & 29,24 & $2,00 \mathrm{a}$ & 1 \\
\hline Midsweet & $200 \mathrm{a}$ & $110 \mathrm{a}$ & $54 \mathrm{a}$ & $12,75 \mathrm{a}$ & $73,65 a$ & $67,64 b$ & 4,18 & 273,72 & $3,12 \mathrm{a}$ & 1 \\
\hline Natal & $260 \mathrm{~b}$ & $130 \mathrm{a}$ & $49 \mathrm{~b}$ & $7,00 \mathrm{~b}$ & $80,90 \mathrm{a}$ & $76,58 \mathrm{a}$ & 4,88 & 28,15 & $3,50 \mathrm{a}$ & 1 \\
\hline Salustiana & $180 \mathrm{a}$ & $80 \mathrm{~b}$ & $61 \mathrm{a}$ & $2,25 \mathrm{c}$ & $65,31 \mathrm{~b}$ & $63,20 \mathrm{~b}$ & 3,20 & 248,17 & $3,12 \mathrm{a}$ & 1 \\
\hline $\mathrm{CV}(\%)$ & 21,46 & 22,00 & 9,79 & 47,48 & 8,61 & 8,67 & - & - & 56,56 & - \\
\hline
\end{tabular}

*Médias seguidas pelas mesmas letras na coluna não diferem entre si, pelo teste Scott-Knott,a 1\% de probabilidade. CV: Coeficiente de variação; M+G: Média e grupo; MF: Massa do fruto; MS: Massa do suco; RS: Rendimento do suco; NS: Número de sementes; DTF: Diâmetro transversal do fruto; AF: Altura do fruto; EP EM: Espessura do epicarpo+ mesocarpo; EEN: Espessura do endocarpo; CEP: Coloração do epicarpo; FF: Formato do fruto;1- Esferoide; 2- Elipsoide.

TABELA 4- Médias dos caracteres qualitativos dos frutos da coleção de citros, Brejão - PE, 2011.

\begin{tabular}{lccccc}
\hline Variedade & SS $\left({ }^{\mathbf{}} \mathbf{B r i x}\right)$ & $\mathbf{p H}$ & AT $(\%$ de a. c.) & Vit. C (a. a.) & Razão SS/AT \\
\hline Laranjas & $\mathbf{M}+\mathbf{G}$ & $\mathbf{M}+\mathbf{G}$ & $\mathbf{M}+\mathbf{G}$ & Médias & M+G \\
Bahia 101 & $10,35 \mathrm{a}$ & $3,50 \mathrm{c}$ & $1,11 \mathrm{a}$ & 34,88 & $10,07 \mathrm{~b}$ \\
Baianinha 03 & $9,00 \mathrm{c}$ & $3,56 \mathrm{c}$ & $0,88 \mathrm{a}$ & 36,01 & $12,38 \mathrm{~b}$ \\
Hamlin 02 & $9,02 \mathrm{c}$ & $3,97 \mathrm{~b}$ & $0,71 \mathrm{a}$ & 25,16 & $9,56 \mathrm{~b}$ \\
Lima & $9,42 \mathrm{~b}$ & $5,81 \mathrm{a}$ & $0,11 \mathrm{~b}$ & 35,24 & $77,61 \mathrm{a}$ \\
Rubi & $9,01 \mathrm{c}$ & $3,51 \mathrm{c}$ & $1,30 \mathrm{a}$ & 48,65 & $8,26 \mathrm{~b}$ \\
Sunstar & $9,01 \mathrm{c}$ & $3,71 \mathrm{~b}$ & $0,96 \mathrm{a}$ & 39,42 & $8,86 \mathrm{~b}$ \\
Pineapple & $9,12 \mathrm{c}$ & $3,72 \mathrm{~b}$ & $1,05 \mathrm{a}$ & 51,99 & $9,53 \mathrm{~b}$ \\
Pera D6 & $9,02 \mathrm{c}$ & $3,51 \mathrm{c}$ & $1,04 \mathrm{a}$ & 30,16 & $9,52 \mathrm{~b}$ \\
Pera D9 & $9,00 \mathrm{c}$ & $3,50 \mathrm{c}$ & $1,19 \mathrm{a}$ & 69,71 & $8,70 \mathrm{~b}$ \\
Pera D12 & $8,40 \mathrm{~d}$ & $3,59 \mathrm{c}$ & $0,96 \mathrm{a}$ & 31,98 & $9,78 \mathrm{~b}$ \\
Valência Tuxpan & $9,10 \mathrm{c}$ & $3,27 \mathrm{c}$ & $1,15 \mathrm{a}$ & 33,88 & $6,96 \mathrm{~b}$ \\
Westin & $9,47 \mathrm{~b}$ & $3,64 \mathrm{~b}$ & $0,85 \mathrm{a}$ & 38,73 & $13,06 \mathrm{~b}$ \\
Midsweet & $9,02 \mathrm{c}$ & $3,22 \mathrm{c}$ & $1,04 \mathrm{a}$ & 40,16 & $9,58 \mathrm{~b}$ \\
Natal & $8,00 \mathrm{~d}$ & $3,90 \mathrm{~b}$ & $0,71 \mathrm{a}$ & 31,47 & $10,16 \mathrm{~b}$ \\
Salustiana & $8,00 \mathrm{~d}$ & $3,66 \mathrm{~b}$ & $0,82 \mathrm{a}$ & 42,63 & $11,74 \mathrm{~b}$ \\
CV(\%) & 3,99 & 4,89 & 31,30 & - & 22,36 \\
\hline
\end{tabular}

*Médias seguidas pelas mesmas letras na coluna não diferem entre si, pelo teste Scott-Knott, a 1\% de probabilidade. CV: Coeficiente de variação; M+G: Média e grupo; SS: Sólidos Solúveis, expresso em ${ }^{\circ}$ Brix; pH: potencial hidrogeniônico; AT: Acidez Titulável; a. c.: ácido cítrico; Vit. C: Vitamina C; a.a.: ácido ascórbico; Razão SS/AT. 


\section{CONCLUSÕES}

1-As variedades de laranjeiras Baianinha 03, Hanlim 02, Pineapple e Pera D6 sobre o porta-enxerto limão Volkameriano-tangerina Cleopatra-264, Midsweet e Westin, apresentam boa qualidade dos frutos, conforme Programa Brasileiro para Modernização da Horticultura, podendo, assim, ser exploradas em condições edafoclimáticas semelhantes ao desenvolvido no experimento.

2-As laranjeiras Pineapple, Valência Tuxpan, Salustiana, Sunstar e Natal são de maturação em meia-estação.

3-Recomenda-se a continuação dos estudos até a estabilização da produção, considerando que as plantas estão em fase de desenvolvimento.

\section{REFERÊNCIAS}

AGUSTI, M.; ZARAGOZZA, S.; BLEIHOLDER, H.; BUHR, L.; HACK,H.; KLOSE, R.; STAUB, R. Adaptation de $1^{\prime}$ échelle $\mathrm{BBCH}$ à la description des stades phénologiques des agrumes du genre Citrus. Fruits, Paris, v.52, n.5, 287-295, 1997.

AOAC - Association of Official Analytical Chemistry. Official methods of analysis. $17^{\text {th }}$ ed. Arlington, 2005. 1928p.

APAC - Agência Pernambucana de Águas e Climas. 2012. Disponível em: htpp:<//www.apac.pe.gov.br.> . Acesso em: 15 jul. 2012.

COUTO, M. A. L.; CANIATTI-BRAZACA, S. G. Quantificação de vitamina $\mathrm{C}$ e capacidade antioxidante de variedades cítricas. Ciência e Tecnologia de Alimentos, Campinas, V.30, n.1, p. 15-19, 2010. Suplemento.

CORREAA, I. M.; MELO, R. da C.; YAMASHITA, R. Y.; RAMOS, H. H. Modificação da escada visando a melhoria da segurança na colheita manual de citros. Revista Brasileira de Agrociência, Pelotas, v. 15, n.1, p. 109-114, 2009.

DOMINGUES, E. T.; TULMANN NETO, A.; TEÓFILO SOBRINHO, J.; MATTOS JUNIOR, D.; POMPEU JUNIOR, J.; FIGUEIREDO, J. O. Seleção de variedades de laranja quanto à qualidade do fruto e período de maturação. Laranja, Cordeirópolis, v.24, n.2, p.471-470, 2003.
DONADIO, L. C.; MOURÃO FILHO, F. A. A.; MOREIRA, C. S. Centros de origem, distribuição geográfica das plantas cítricas e histórico da citricultura no Brasil. In: MATTOS JÚNIOR, D.; NEGRI, J.D.; PIO, R.M.; POMPEU JUNIOR, J. Citros. Campinas: Instituto Agronômico e FUNDAG, 2005. cap.1, p.1-18.

FAO. Production of orange. Disponível em: $<\underline{\text { http:// }}$ faostat.fao.org/default.aspx $>$. Acesso em: 6 jul. 2012.

FIDALSKI, J.; SCAPIMC. A.; STENZEL, N. M. C. Divergence of 'folha murcha' orange tree rootstocks as influenced by two groundcover crops Revista Brasileira de Ciência do Solo, Viçosa, MG, v.31, n.2, p 353-360 2007.

IBGE. Produção agrícola municipal: culturas perenes. Disponível em: $<\underline{\mathrm{http}}$ :/Www.ibge.gov.br $>$. Acesso em: 6 jul. 2012.

INMET - Instituto Nacional de Meteorologia. Agricultura: balanço hídrico climático. Disponível em: $<$ http://www.inmet.gov.br/html/agro.html $>$. Acesso em: 14 jul. 2012.

IPGRI - International Plant Genetic Resources Institute Rome. Descriptors for citrus. Rome, 1999. $27 \mathrm{p}$.

MACHADO, M. A.; YALY-C.,M.; BATIANEL, M. Melhoramento, genética e genômica de citros para resistência a doenças. Revista Brasileira de Fruticultura, Jaboticabal, v. 33, n.1 - edição especial, p.158-172.

MACHADO, M.A.MENDEL, K. Rootstock-scion relationships in Shamouti trees on light soil. Ktavim, Rehovot, v.6, p.35-60, 1956.

MUNHOZ, J. R.; MORABITO, R. Otimização no planejamento agregado de produção em indústrias de processamento de suco concentrado congelado de laranja. Gestão de Produção, São Carlos, v.17, n.3, p.465-481, 2010.

NEGREIROS, J. R. da S.; LESSA, L. S.; RONCATTO, G.; RODRIGUES, M. J. da S.; ALBUQUERUE, M. F.; LOPES, L. M.; SOARES FILHO, W. dos S. Caracterização agronômica de diferentes porta-enxertos cítricos enxertados sob laranja pêra. In: CONGRESSO BRASILEIRO DE FRUTICULTURA, 21., 2010, Natal. Anais... Natal: Sociedade Brasileira de Fruticultura, 2010. 
NEVES, M. F.; TRONBIM, V. G.; MILAU, P.; LOPES, F. F.; CRESSONI, F.; KALAKI, R. Citricultura brasileira. Ribeirão Preto: FEA/USP, 2010. p.137.

OLIVEIRA, R. P. de; SCHRODER, E. C.; SOUZA, E. L. de S.; SCIVITTARO, W. B.; CASTRO, L. A. S. de.; ROCHA, P. S. G. da Laranjeiras sem acidez. Pelotas: Embrapa Clima Temperado Pelotas, 2010. (Documento, 298).

PASSOS, O. S.; SOUSA, C. A. F.; SOARES FILHO, W. S. Alternativas de porta-enxertos de citros no Nordeste do Brasil. Cruza das Almas: Embrapa Mandioca e Fruticultura Tropical, 2005. 6 p. In: POMPEU JÚNIOR, J.; BLUMER, S., Laranja, Cordeirópolis, v.27, n.2, p.341-354, 2006.

PELLEGRINI, N. B.;COLOMBI; SALVATORE S.; BRENNA, O. V.; GALAVERNA, G.; DEL RIO, D.; BIANCHI, M.; BENNETT, R. N.; BRIGHENTI, F. Evaluation of antioxidant capacity of some fruit and vegetable foods: efficiency of extraction of a sequence of solvents. Journal of the Science of Food and Agriculture, London, v. 87, n. 1, p. 103111, 2007.

PEREIRA; M. E. C.; CANTILLANO F. F.; GUTIEREZ, A. S. D. ALMEIDA G. V. B. Procedimentos pós-colheita na produção integrada de citros. Cruz das almas: Embrapa Mandioca e Fruticultura Tropical, 2006. p.40. (Documentos, 156).

POMPEU JUNIOR, J. Porta-enxertos. In: MATTOS JUNIOR, D.; DE NEGRI, J. D.; PIO, R. M.;POMPEU JUNIOR, J. (Ed.). Citros. Campinas: Instituto Agronômico e Fundag, 2005. p. 61-104.

POMPEU JUNIOR J.; BLUMER, S.; GARCIA, V. X. P. Características dos frutos das laranjeiras Gardner, Midsweet e Sunstar. Laranja, Cordeirópolis, v.30, n.1-2, p.65-73, 2009.
PROGRAMA BRASILEIRO PARA MODERNIZAÇÃO DA HORTICULTURA. Normas de classificação de citros de mesa. São Paulo: CEAGESP, 2011. p.12.

SACRAMENTO, C. K.; COELHO, I. S.; ALDAR, T. Maturação e qualidade das laranjas (Citrus sinensis) 'Bahia', 'Pêra', 'Natal' e 'Valência' na região dos Tabuleiros do Sul da Bahia. Revista Agrotrópica, Itabuna, v.1, n.3, p.198-203, 1988.

SARTORI, I. A.; KOLLER, O. C.; SCHWARZ, S. F.; BENDER, R. J.; SCHAFER, G. Maturação de frutos de seis cultivares de laranjas doces na depressão central do Rio grande do Sul. Revista Brasileira de Fruticultura, Jaboticabal, v.24, p.364-369, 2002.

SCHINOR, E. H.; AGUILAR-VILDOSO, C. I.; MOURÃO FILHO, F. DE A. A. Caracterização agronômica de seleções de laranjeira Pêra e sua relação com a mancha preta dos citros. Laranja, Cordeirópolis, v.30, n.1-2, p.75-96, 2009.

SECRETARIA DE CIÊNCIA, TECNOLOGIA E MEIO AMBIENTE DE PERNAMBUCO. Atlas de bacias hidrográficas de Pernambuco. Recife, 2006. 104p.

SOUZA, P. V. D. DE; SALDANHA, E. L. DE; OLIVEIRA, R. P. DE; BONINE, D. P. Indicações técnicas para a citricultura no Rio Grande do Sul. Porto Alegre: FEPAGRO, 2010. p.126.

STENZEL, N. M. C.; NEVES, C. S. V. J.; MARUR, C. J.; GOMES, J. C. Crescimento Vegetativo de Plantas Cítricas no Norte e Noroeste do Paraná. Revista Brasileira de Fruticultura, Jaboticabal, v. 27, n.3, p.412-417, 2005.

STENZEL, N. M. C.; NEVES, C. S. V. J.; GONZALEZ, M. G. N.; SCHOLZ, M. B. dos S.; GOMES, J. C. Desenvolvimento vegetativo, produção e qualidade dos frutos da laranjeira 'Folha Murcha' sobre seis porta-enxertos no Norte do Paraná. Ciência Rural, Santa Maria, v.35, n.6, p. 1281-1286, 2005.

WESTPHALEN, F. Citricultura. Rio Grande do Sul: Editora da Universidade Federal de Santa Maria, 2008. p.02-05. 